\title{
Total Hip Arthroplasty for Secondary Coxarthrosis in Patients with Hereditary Multiple Exostoses: Minimum 5-Year Follow-up Results and Surgical Considerations
}

\author{
Jae Youn Yoon, MD, Chan-Woo Park, $\mathrm{MD}^{*}$, Youn-Soo Park, $\mathrm{PhD}$, \\ Jeong Joon Yoo, $\mathrm{PhD}^{\dagger}$, Hee Joong Kim, $\mathrm{PhD}^{\dagger}$ \\ Department of Orthopedic Surgery, Dongguk University Ilsan Hospital, Goyang, \\ *Department of Orthopedic Surgery, Samsung Medical Center, Sungkyunkwan University School of Medicine, Seoul, \\ ${ }^{\dagger}$ Department of Orthopedic Surgery, Seoul National University College of Medicine, Seoul, Korea
}

Background: Hereditary multiple exostoses (HME) is an autosomal dominant disorder. The lesion in the proximal femoral metaphysis can bring about hip dysplasia and subsequent degenerative arthritis. Due to its rare prevalence, there have been a few case reports of total hip arthroplasty (THA) for osteoarthritis secondary to HME. The aim of this study was to report mid- to long-term outcomes of THA in HME patients and discuss special considerations that should be taken into account during surgery.

Methods: We retrospectively evaluated the clinical and radiological results of THA for osteoarthritis secondary to HME in 11 hips of 9 patients after a minimum follow-up of 5 years (mean, 9.9 years). There were 3 men ( 3 hips) and 6 women (8 hips), with a mean age of 53.6 years (range, 46.8-58 years) at the index surgery in this study. Harris hip score (HHS) was used for clinical outcome assessment, and radiologically, implant stability, radiolucent lines, liner wear, and any sign of osteolysis or implant loosening were evaluated. Postoperative complications including infection, deep vein thrombosis, and dislocations were also investigated.

Results: Cemented stems and cementless cups with the conventional polyethylene liner were used in bilateral hips of a single patient. In the other cases, cementless implants were used with ceramic-on-ceramic bearings. The mean HHS improved from 34.8 preoperatively to 92.5 postoperatively. Polyethylene liner wear and osteolysis were observed in 1 patient with cemented stems. Radiolucent lines were observed in 2 different cases. However, the femoral stems remained stable. There were no surgery-related complications except heterotopic ossification during follow-up.

Conclusions: Despite the several surgical considerations, the mid- to long-term clinical and radiological outcomes of THA in HME patients were satisfactory. The abnormal, wide mediolateral diameter of the proximal metaphysis should be considered in selecting and inserting the stem with adequate anteversion. Leg length discrepancy was also common, so teleradiographs should be obtained before surgery. Intraoperative leg length evaluation might be difficult due to the morphologic changes in the proximal femur after mass excision and individual bone length differences.

Keywords: Hip joint, Multiple hereditary exostoses, Osteochondromas, Osteoarthritis, Total hip arthroplasty

Received March 4, 2020; Accepted March 25, 2020

Correspondence to: Hee Joong Kim, PhD

Department of Orthopedic Surgery, Seoul National University College of

Medicine, 103 Daehak-ro, Jongno-gu, Seoul 03080, Korea

Tel: +82-2-2072-2970, Fax: +82-2-764-2718

E-mail: oskim@snu.ac.kr
Hereditary multiple exostoses (HME) is a rare, autosomal dominant inherited disease, with an approximate incidence of 0.9 to 2.0 in 100,000 individuals. ${ }^{1-3)}$ HME usually affects the epiphysis of long bones, resulting in abnormal chondro-osseous growth around the metaphysis. ${ }^{4)}$ In regard to malignant changes, reported proportions varied 
from less than $1 \%$ to $7 \%$ in recent large-scale cohort studies, and if a malignant change is suspected, surgical excision should be considered. ${ }^{5-7)}$

The most common sites for the occurrence of HME are the distal femur, the proximal humerus, and the proximal tibia. The proximal femur is also reported to be involved commonly, with an approximate prevalence of $30 \%$ to $90 \%{ }^{8)}$ Compared to lesions occurring at the superficial joints, such as the knee, shoulder, or elbow, lesions growing around the hip joint rarely cause hip pain or other symptoms in younger ages. Hip joint osteochondromas usually present with deformities and premature osteoarthritis (OA) ${ }^{2,8,9)}$ Moreover, patient's symptoms may differ depending on the location of the lesion. Lesions growing in the lateral aspect of the femur usually cause pain or discomfort but lesions in the medial side of the proximal femur or near the acetabulum may alter the normal hip anatomy, causing coxa valga, acetabular dysplasia, and subluxation of the femoral head. ${ }^{10)}$ These deformities eventually lead to secondary OA and chronically worsen the patient's social and psychological well-being as well as functional abilities (Fig. 1). ${ }^{11)}$

Although there have been various surgical attempts to preserve affected hip joints in patients with no or minimal arthritic changes, total hip arthroplasty (THA) is indicated in patients with severe pain and dysfunction due to advanced hip OA. However, mainly due to the rare prevalence, there are only a few case reports regarding author's personal experience on THA for OA secondary to a proximal femoral lesion of HME. Furthermore, surgical concerns related to the extent of mass excision, proper stem selection for the bizarre-shaped proximal femur, and individual bone length discrepancy have not been dealt with well. We believe that THA could be a safe and effective treatment for symptomatic HME patients if the variables are sufficiently considered, and since 1995, THA has been performed in HME patients with severe hip pain and activity restrictions. The objective of our study was to evaluate the clinical and radiological outcomes of THA in patients with HME and advanced hip OA after a minimum follow-up of 5 years and present our experience with special considerations regarding this patient group.

\section{METHODS}

\section{Study Design and Patient Selection}

This is a retrospective case-series study conducted at 2 different institutions. This study was conducted in compliance with the principles of the Declaration of Helsinki and was approved by Institutional Review Board of Seoul National University Hospital (IRB No. H-1803-028-927) and Samsung Medical Center (IRB No. 2020-07-019). The informed consent was waived.

We reviewed medical data and radiographic images of the patients who underwent THAs due to HME between December 1995 and May 2011. Fourteen primary THAs were performed in 11 consecutive patients with HME because of painful hip pain and OA secondary to the proximal femoral lesions around the hip joint. Three cases (2 patients) were excluded from this study because of the short follow-up period (2.1 years, 2.8 years, and 3.7 years each) and 11 cases (9 patients) were included in the final analysis. There were 3 men ( 3 hips) and 6 women ( 8 hips), with a mean age of 53.6 years (range, $46.8-58$ years) at the index surgery. The mean height and weight were $156.3 \mathrm{~cm}$ (range, 145-174.6 cm) and 62.4 kg (range, 48-75.7 kg), respectively. The mean duration of follow-up was 9.9 years (range, 5.1-20.4 years).
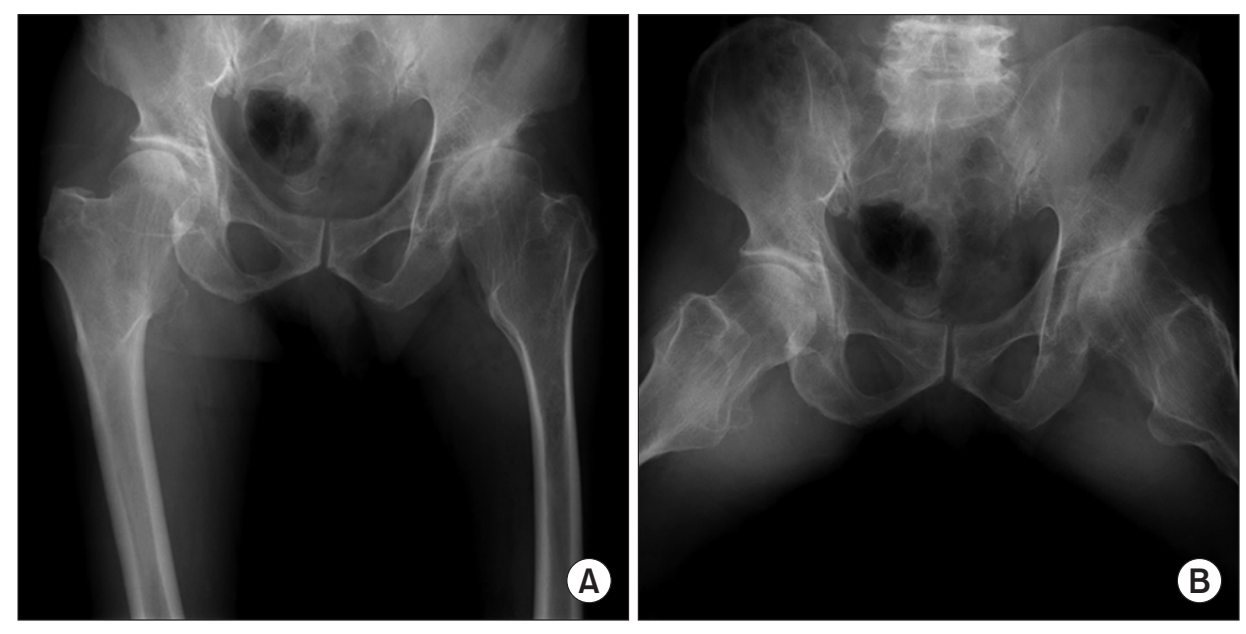

Fig. 1. A 56-year-old male patient with bilateral hip involvement. Anteroposterior (A) and frogleg (B) images show coxa valga deformity and acetabular dysplasia with marked degenerative changes in the left hip. Both the femoral neck and intertrochanteric area are wide not only anteroposteriorly but also mediolaterally. 
Yoon et al. Total Hip Arthroplasty for Coxarthrosis in Hereditary Multiple Exostoses

Clinics in Orthopedic Surgery • Vol. 12, No. 4, $2020 \bullet$ www.ecios.org

\section{Surgical Procedures}

All surgical procedures were performed by 2 senior surgeons (YSP and HJK) at each institution. Two patients underwent bilateral THAs, and 1 of them was treated with cemented stems using metal heads (Orthinox, Stryker, Mahwah, NJ, USA) and ultra-high-molecular-weight polyethylene (ABG II, Stryker) bearing couple. The other 8 patients ( 9 cases) were treated with cementless stems using a ceramic-on-ceramic bearing couple (Biolox Forte or Delta; Ceramtec, Plochingen, Germany).

Surgery was performed in the lateral decubitus position through a modified Hardinge's anterolateral approach (5 hips in 5 patients), a modified Gibson's posterolateral approach (4 hips in 3 patients), or a transtrochanteric approach ( 2 hips in 1 patient). During the procedures, tumorous lesions were removed as much as possible and some portion of the remnant femoral neck was removed using an osteotome and a rongeur when it caused impingement limiting the range of hip motion. Intraoperatively, a simple radiograph was taken after the trial reduction to check the implant position and leg length in most cases.

Implants used in the operation and other surgical details are presented in Table 1. The Exeter femoral stem is a double-tapered, polished, collarless, cemented stem, which has proven its good long-term survival with low revision rates. ${ }^{12)}$ The S-ROM femoral prosthesis (Depuy, Warsaw, IN, USA) is a proximally modular cementless stem with adjustable offsets and femoral versions. The Bicontact SD stem (Aesculap, Tuttlingen, Germany) is a cementless titanium-alloy stem with a slightly tapered, rectangular, collarless design. Both the modified Coren stem and Bencox II stem (Corentec, Seoul, Korea) are doubletapered wedge, rectangular, collarless stems and have a grip-blasted surface treated with micro arc oxidation to maximize bone ongrowth. There is no morphologic difference between the 2 stems and only the trademarked names differ. Finally, the Bencox ID stem (Corentec) is a singlewedged cementless prosthesis with a double-tapered pressfit. It has a titanium-plasma sprayed porous coating at the proximal $5 / 8$ of the implant. Meanwhile, all acetabular cups were fixed to the pelvis by using press-fit method, and no cement, allogenic bone graft, or metal augment were used during surgery.

After surgery, ordinary postoperative protocols were applied in maintaining hip position and gradual weightbearing using crutches was initiated. After discharge, patients were examined clinically and radiologically at 6 weeks, 3 months, 9 months, and then annually after surgery. Clinical outcomes were assessed using a modified
Harris hip score (HHS) and a questionnaire regarding thigh pain and noise. For clinical results, a total HHS below 70 points was considered poor, 70 to 79 points reasonable, 80 to 89 points good, and 90 to 100 points excellent. Man-Whitney test was used for statistical analysis.

Radiologically, component stability, radiolucent lines, liner wear, osteolysis, migration or loosening of the component, and heterotopic ossification (HO) were checked on serial radiographs. We additionally measured the canal to calcar isthmus ratio and Sharp's acetabular angle to evaluate the degree of accompanied acetabular dysplasia. ${ }^{13,14)}$ When radiographically measured, 9 hips (82\%) had acetabular dysplasia. The zones described by Gruen et al. ${ }^{15)}$ and those described by DeLee and Charnley ${ }^{16)}$ were used to assess the location and extent of the radiolucent line and osteolysis. When a $\mathrm{HO}$ was observed, it was graded by the criteria of Brooker et al. ${ }^{17)}$ Other complications, such as dislocation, infection, and deep vein thrombosis, and any possibility of malignant transformation, were also investigated.

\section{Statistical Analysis}

Patient's clinical outcome was marked as a mean value with range, and Student test with a probability level of 0.05 was used for analysis. Statistical analysis was performed by using IBM SPSS ver. 21 (IBM Corp., Armonk, NY, USA).

\section{RESULTS}

\section{Clinical Results}

There was no case complicated by dislocation, infection, or deep vein thrombosis. The mean HHS improved from 34.8 (range, 24-50) preoperatively to 92.5 (range, 83-100) at the latest follow-up $(p<0.001)$. Seven hips were rated as excellent (64\%) and 4 hips were rated as good (36\%). No patients complained of thigh pain or noise, including the squeaking or clicking sound.

\section{Radiographic Results}

Preoperatively, the patients' radiographic images showed broad and blunt metaphyses with various degrees of hip dysplasia and subluxation. The mean canal to calcar isthmus ratio was $34.6 \%$ (range, $24 \%-50.7 \%$ ). Nine hips (82\%) were classified as Dorr type A and only 2 hips (18\%) were classified as Dorr type B according to the Caput to Collum ratio. ${ }^{13)}$ Sharp's acetabular angle ranged from $41.5^{\circ}$ to $51.7^{\circ}$ with the mean value of $45.9^{\circ}$, and the extrusion index ranged from 0.14 to 0.43 with a mean value of $0.32^{\circ} .^{14)}$ On the immediate postoperative radiographs, all implants were found to be stably fixed. There was no case of implant 
Yoon et al. Total Hip Arthroplasty for Coxarthrosis in Hereditary Multiple Exostoses

Clinics in Orthopedic Surgery • Vol. 12, No. 4, $2020 \bullet$ www.ecios.org

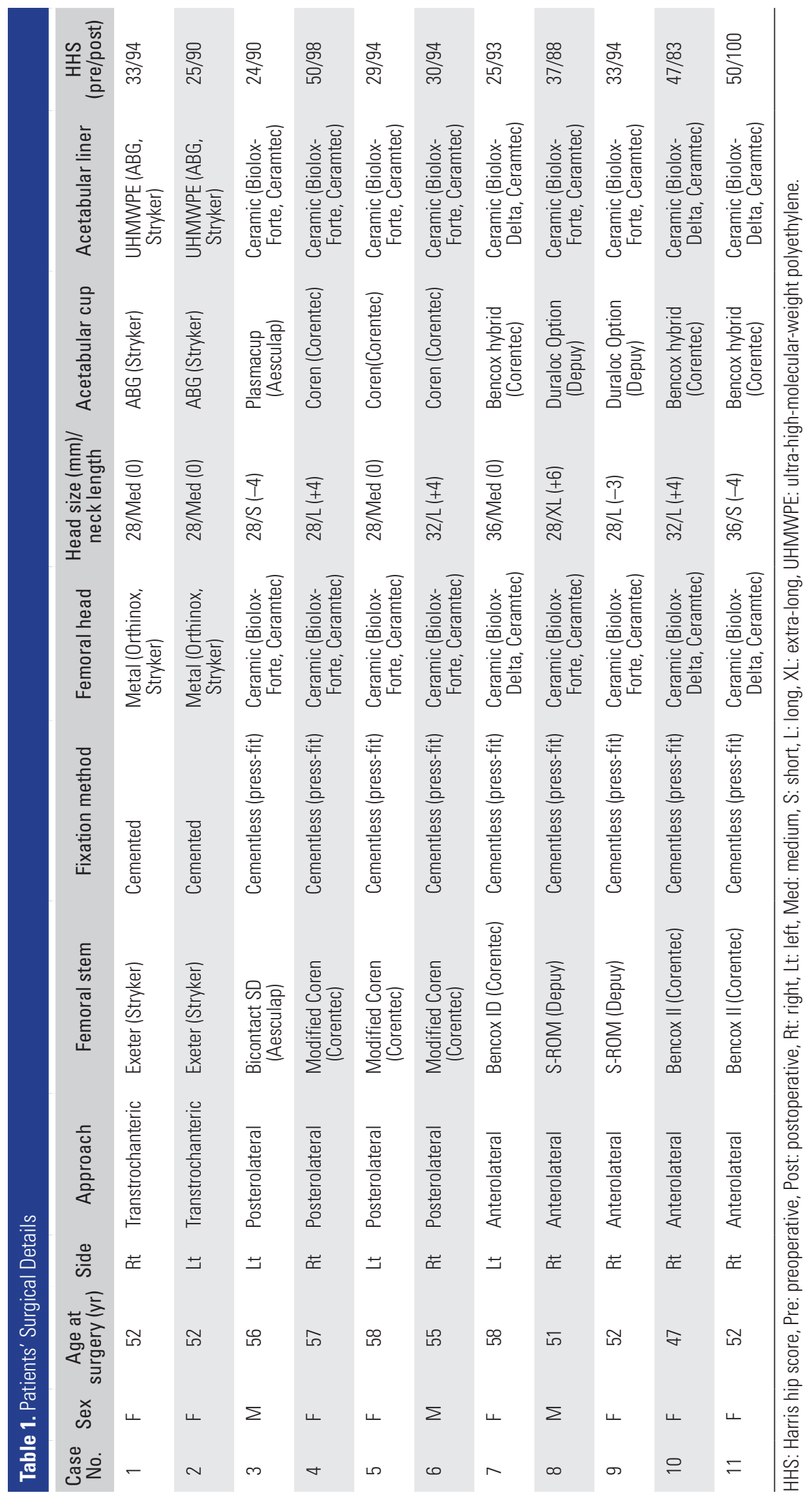


loosening or periprosthetic fracture, and revision surgery was not necessary till the last follow-up. Radiolucent lines were detected around the femoral stem in 2 hips ( 2 patients, case 6 and 9). In 1 case in which a modified Coren stem was used (case 6), the radiolucent lines were mainly located in Gruen zones 1 and 7 and in the upper half of the zones 2 and 6 (Fig. 2A). ${ }^{15)}$ The other case (case 9) had radiolucent lines in Gruen zones 4 and 5 around the S-ROM modular stem (Fig. 2B), which has porous-coating only in the proximal sleeve to achieve bony ingrowth. Radiolucent lines were not detected around the metal shell in any case. Liner wear was observed in 2 hips (1 patient, case 1 and 2) in which a conventional polyethylene liner and metal head were used. Periacetabular osteolysis was also present in 1 hip (case 1) in the DeLee and Charnley zone I (Fig. 3). ${ }^{16}$ On the latest follow-up examination at postoperative 20.4 years, both hips were asymptomatic, without evidence of implant loosening. The wear and osteolysis were not rapidly progressive. HO was detected in 4 out of 11 hips (36\%). Three cases were Brooker grade 1, and 1 case (case 4) was grade $3 \mathrm{HO}$, but no case was symptomatic. ${ }^{17)}$

\section{DISCUSSION}

Involvement of the proximal femur in HME is relatively common, with a reported incidence of $30 \%$ to $90 \%$, and unlike other superficial joints, hip osteochondromas are deep-seated and hardly palpated in the early decades of
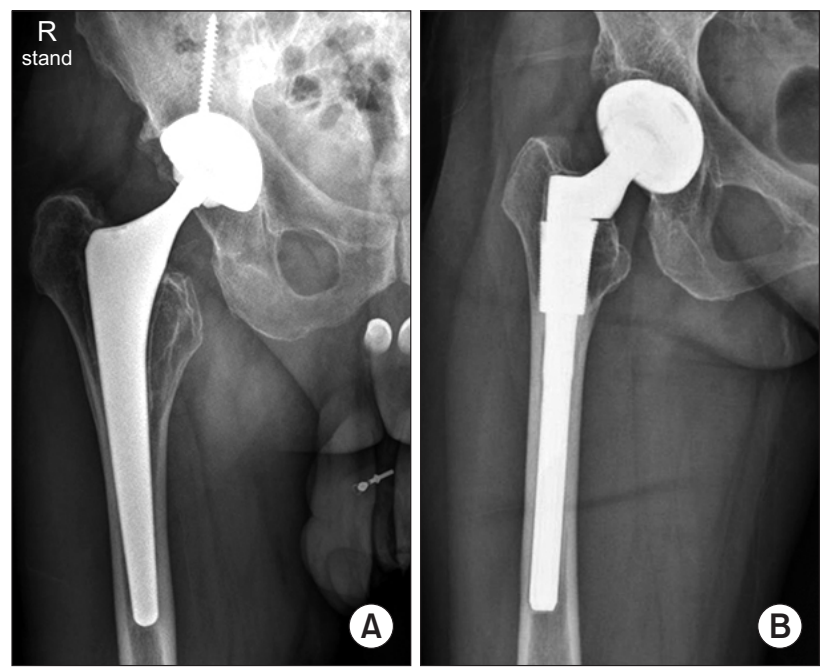

Fig. 2. Radiolucent lines around the femoral stem were observed in 2 cases during clinical follow-up. (A) An anteroposterior radiograph taken at 4 years postoperatively shows radiolucent lines in Gruen zones 1 and in the upper half of zones 2 and 6. (B) An anteroposterior radiograph taken 6.2 years postoperatively demonstrates radiolucent lines in Gruen zones 4 and 5 . life. ${ }^{2,8,18,19)}$ At the time of diagnosis, bony deformities and early OA are often present. ${ }^{2,8,9)}$ Although recent papers have reported a less common incidence of acetabular dysplasia, approximately $25 \%$ of patients with HME in the hip joint had various degrees of coxa valga and acetabular dysplasia. ${ }^{2021)}$ These skeletal dysplasias, along with the exophytic bone mass itself, may induce subluxation of the femoral head. ${ }^{9)}$ In this study, however, the mean Sharp's acetabular angle was $45.9^{\circ}$ (normal value, $38^{\circ}-42^{\circ}$ ) and the mean extrusion index was 32\% (normal value, 17\%-26\%). Acetabular dysplasia was diagnosed according to the radiological criteria of Jacobsen et al. ${ }^{22)}$ and Sharp ${ }^{14)}$ in 8 hips (73\%) and 9 hips (82\%), respectively. Both the proportion and the severity of the acetabular dysplasia were higher in this patient group. This difference may be attributable to the selection of the patients who were all diagnosed with premature OA of the hip. However, the degree of undercoverage was not severe enough to require allogenic bone graft or metal augmentation, and acetabular cups were well fixed with the press-fit method.

Acetabular dysplasia, subluxation of the femoral head, coxa valga, and wide metaphyses are deformities frequently seen with an exostosis in the proximal femur. The femoral stem implant choice is important for filling of a wide metaphysis. In previous case reports, cemented stems or modular type cementless stems were used because the metaphysis was wide not only mediolaterally but also anteroposteriorly (Fig. 1). ${ }^{23-26)}$ Among the nonmodular type cementless stems used in this study, the modified Coren stem, renamed as the Bencox II stem later, was straight, double wedged, and tapered with rectangular cross-section. Its whole surface was grit-blasted but its proximal portion was relatively narrow. This stem was

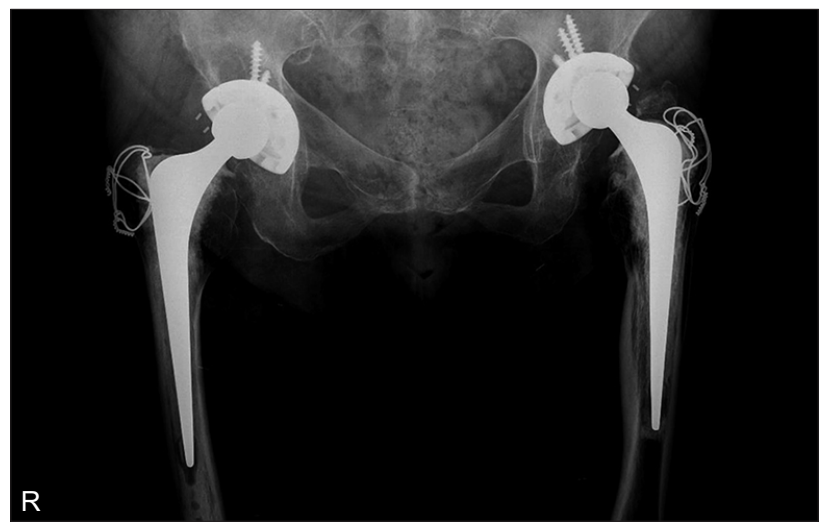

Fig. 3. An anteroposterior radiograph of a female patient (case 3) taken 11 years postoperatively shows polyethylene liner wear in both hips. Periacetabular osteolysis is observed in the right hip mainly in the DeLee and Charnley zone 1. 
Yoon et al. Total Hip Arthroplasty for Coxarthrosis in Hereditary Multiple Exostoses

Clinics in Orthopedic Surgery • Vol. 12, No. 4, $2020 \bullet$ www.ecios.org

used in 5 cases. Out of them, radiolucency was observed in Gruen zones 1, 2, 6, and 7 in 1 case (Fig. 2A). The width of the radiolucency was wider proximally. This finding was an unusual one compared with other reported results of the implant and highlighted the importance of implant selection for cases with wide metaphysis. ${ }^{27,28)}$ Coxa valga, together with an increased anteversion, was also a frequent deformity, but it did not cause technical difficulty because the femoral neck was also wide mediolaterally and anteroposteriorly. However, it was necessary to keep the correct version of the femoral rasps, since the wide anteroposterior diameter of the proximal femoral canal could alter the anatomical guidance of the femoral stem to its native shape and bring confusion to the surgeon in determining the adequate femoral anteversion. ${ }^{18,19)}$

In 1 case, there was difficulty in adjusting the leg length intraoperatively. An intraoperative radiograph was taken after a trial reduction, but the shape of the proximal femur was changed markedly after removal of the large irregularly shaped tumor mass. It was impossible to compare the preoperative and intraoperative findings. We tried to compare the knee level, but the difference was unexpectedly large. As a result, the neck length was decided based only on the intraoperative assessment of joint stability, which was not reliable because of the resulting laxity after removal of the tumor mass and prominent metaphysis to avoid impingement. After surgery, we found that the individual bone length of both legs were quite different. The femur of the operation side was much longer than that of the contralateral side and the reverse was true for the tibia resulting in the same total leg lengths (Fig. 4). Leg length differences (LLD) are a frequent finding in HME, with a prevalence of $8 \%$ to $25 \% .{ }^{2,18,29)}$ Noonan et al. ${ }^{30)}$ reported that the mean LLD was approximately $1.8 \mathrm{~cm}$ (range, $0.5-5.5 \mathrm{~cm}$ ) and that there were individual bone length differences in some cases. For HME cases, it is mandatory to check the leg length on a teleradiograph preoperatively.

There were several limitations in this study. First, the number of cases might not have been large enough for analysis of the results of the THAs. However, the incidence

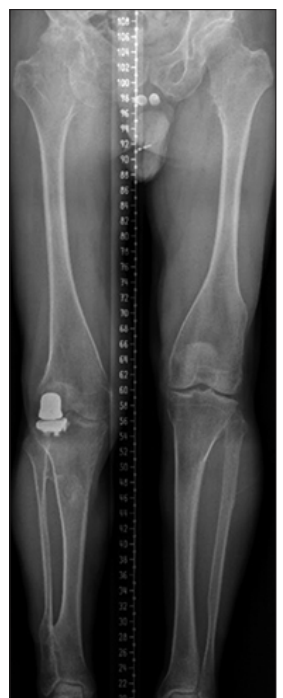

Fig. 4. A teleradiograph of a male patient showing individual bone length differences in both femurs and tibiae. There is no big difference in total leg length.

of HME is not high and the follow-up period of this study was longer than that of previous reports. Second, it was not a single surgeon's series and different surgical methods and implants of various designs were used. However, both surgeons were experienced hip surgeons and implant selection was determined based on the skeletal condition of each case with HME.

This study suggests that good results can be achieved in THA for OA secondary to HME using cementless stems properly selected according to the geometry of the proximal femur. It emphasizes the necessity of preoperative teleradiographs and possible difficulties in intraoperative leg length evaluation due to joint laxity after removal of the tumor mass and wide metaphysis to avoid impingement.

\section{CONFLICT OF INTEREST}

No potential conflict of interest relevant to this article was reported.

\section{REFERENCES}

1. Clement ND, Duckworth AD, Baker AD, Porter DE. Skeletal growth patterns in hereditary multiple exostoses: a natural history. J Pediatr Orthop B. 2012;21(2):150-4.

2. Schmale GA, Conrad EU 3rd, Raskind WH. The natural history of hereditary multiple exostoses. J Bone Joint Surg Am. 1994;76(7):986-92.
3. Thompson RL, Hosseinzadeh P, Muchow RD, et al. Syringomyelia and vertebral osteochondromas in patients with multiple hereditary exostosis. J Pediatr Orthop B. 2014;23:44953.

4. Ryckx A, Somers JF, Allaert L. Hereditary multiple exostosis. Acta Orthop Belg. 2013;79:597-607. 
Yoon et al. Total Hip Arthroplasty for Coxarthrosis in Hereditary Multiple Exostoses

Clinics in Orthopedic Surgery • Vol. 12, No. 4, $2020 \bullet$ www.ecios.org

5. Czajka CM, DiCaprio MR. What is the proportion of patients with multiple hereditary exostoses who undergo malignant degeneration? Clin Orthop Relat Res. 2015;473(7):2355-61.

6. Goud AL, de Lange J, Scholtes VA, Bulstra SK, Ham SJ. Pain, physical and social functioning, and quality of life in individuals with multiple hereditary exostoses in The Netherlands: a national cohort study. J Bone Joint Surg Am. 2012;94(11):1013-20.

7. Pedrini E, Jennes I, Tremosini M, et al. Genotype-phenotype correlation study in 529 patients with multiple hereditary exostoses: identification of "protective" and "risk" factors. J Bone Joint Surg Am. 2011;93(24):2294-302.

8. Sorel JC, Facee Schaeffer M, Homan AS, Scholtes VA, Kempen DH, Ham SJ. Surgical hip dislocation according to Ganz for excision of osteochondromas in patients with multiple hereditary exostoses. Bone Joint J. 2016;98(2):260-5.

9. Porter DE, Benson MK, Hosney GA. The hip in hereditary multiple exostoses. J Bone Joint Surg Br. 2001;83(7):988-95.

10. El-Fiky TA, Chow W, Li YH, To M. Hereditary multiple exostoses of the hip. J Orthop Surg (Hong Kong). 2009;17(2):161-5.

11. D'Ambrosi R, Ragone V, Caldarini C, Serra N, Usuelli FG, Facchini RM. The impact of hereditary multiple exostoses on quality of life, satisfaction, global health status, and pain. Arch Orthop Trauma Surg. 2017;137(2):209-215.

12. Nieuwenhuijse MJ, Valstar ER, Kaptein BL, Nelissen RG. The exeter femoral stem continues to migrate during its first decade after implantation: 10-12 years of followup with radiostereometric analysis (RSA). Acta Orthop. 2012;83(2):129-34.

13. Dorr LD, Faugere MC, Mackel AM, Gruen TA, Bognar B, Malluche HH. Structural and cellular assessment of bone quality of proximal femur. Bone. 1993;14(3):231-42.

14. Sharp IK. Acetabular dysplasia: the acetabular angle. J Bone Joint Surg Br. 1961;43(2):268-72.

15. Gruen TA, McNeice GM, Amstutz HC. "Modes of failure" of cemented stem-type femoral components: a radiographic analysis of loosening. Clin Orthop Relat Res. 1979;(141):1727.

16. JG, Charnley J. Radiological demarcation of cemented sockets in total hip replacement. Clin Orthop Relat Res. 1976;(121):20-32.

17. Brooker AF, Bowerman JW, Robinson RA, Riley LH Jr. Ectopic ossification following total hip replacement: incidence and a method of classification. J Bone Joint Surg Am.
1973;55(8):1629-32.

18. Shapiro F, Simon S, Glimcher MJ. Hereditary multiple exostoses: anthropometric, roentgenographic, and clinical aspects. J Bone Joint Surg Am. 1979;61(6):815-24.

19. Stieber JR, Dormans JP. Manifestations of hereditary multiple exostoses. J Am Acad Orthop Surg. 2005;13(2):110-20.

20. Higuchi C, Sugano N, Yoshida K, Yoshikawa H. Is hip dysplasia a common deformity in skeletally mature patients with hereditary multiple exostoses? J Orthop Sci. 2016;21(3):323-6.

21. Wang YZ, Park KW, Oh CS, et al. Developmental pattern of the hip in patients with hereditary multiple exostoses. BMC Musculoskelet Disord. 2015;16:54.

22. Jacobsen S, Sonne-Holm S, Soballe K, Gebuhr P, Lund B. Hip dysplasia and osteoarthrosis: a survey of 4151 subjects from the osteoarthrosis substudy of the Copenhagen city heart study. Acta Orthop. 2005;76(2):149-58.

23. Annapureddy SR, Chapman TW, Charnley GJ. Dual aetiology hip pain requiring total hip arthroplasty in hereditary multiple exostoses: a case report and review of the literature. Hip Int. 2002;12(4):397-9.

24. Kanda A, Kaneko K, Obayashi O, Mogami A. Total hip arthroplasty using a polished tapered cemented stem in hereditary multiple exostosis. Case Rep Orthop. 2016;2016: 4279060 .

25. Moran M, Krieg AH, Boyle RA, Stalley PD. Bilateral total hip arthroplasty in severe hereditary multiple exostosis: a report of two cases. Hip Int. 2009;19(3):279-82.

26. Vaishya R, Swami S, Vijay V, Vaish A. Bilateral total hip arthroplasty in a young man with hereditary multiple exostoses. BMJ Case Rep. 2015;2015:bcr2014207853.

27. Kim HJ, Yoo JJ, Seo W, Kim MN, Kang T. Cementless total hip arthroplasty using the Coren hip system: a minimum five-year follow-up study. Hip Pelvis. 2018;30(3):162-7.

28. Lee JM, Sim YS, Choi DS. Hip arthroplasty using the Ben$\operatorname{cox}^{\circledR}$ hip system: an evaluation of a consecutive series of one thousand cases. Hip Pelvis. 2018;30(4):210-8.

29. Clement ND, Porter DE. Can deformity of the knee and longitudinal growth of the leg be predicted in patients with hereditary multiple exostoses? A cross-sectional study. Knee. 2014;21(1):299-303.

30. Noonan KJ, Feinberg JR, Levenda A, Snead J, Wurtz LD. Natural history of multiple hereditary osteochondromatosis of the lower extremity and ankle. J Pediatr Orthop. 2002;22(1):120-4. 